\title{
Pneumothorax and pneumomediastinum in patients with COVID-19: A retrospective study from tertiary care institute in India
}

\author{
Saurav Sekhar Paul ${ }^{1}$, Bhavesh Mohan Lal ${ }^{1}$, Animesh Ray,*, Ved Prakash Meena ${ }^{1}$, \\ Rohit Kumar Garg ${ }^{1}$, Pawan Tiwari ${ }^{3}$, Prashant Sirohiya ${ }^{2}$, Saurabh Vig ${ }^{2}$, \\ Sushma Bhatnagar ${ }^{2}$, Anant Mohan ${ }^{3}$, Surabhi Vyas ${ }^{4}$, Naveet Wig ${ }^{1}$ \\ ${ }^{1}$ Department of Medicine, All India Institute of Medical Sciences, New Delhi, India; \\ ${ }^{2}$ Department of Oncoanesthesia and Palliative Medicine, National Cancer Institute, All India Institute of Medical Sciences, Jhajjar, India; \\ ${ }^{3}$ Department of Pulmonary, Critical care and Sleep Medicine, All India Institute of Medical Sciences, New Delhi, India; \\ ${ }^{4}$ Department of Radiodiagnosis and Intervention Radiology All India Institute of Medical Sciences, New Delhi, India.
}

SUMMARY COVID-19 is associated with rarer extra-parenchymal manifestations, namely pneumothorax (PTX) and pneumomediastinum (PM) leading to complications and increased mortality. The study aims to describe the prevalence, risk factors for mortality, radiological characteristics and outcome of PTX/ PM in patients admitted with COVID-19. This was a retrospective, single-centre, observational study in patients with confirmed COVID-19 presenting with non-iatrogenic PTX/PM from April 2020 to May 2021. Details pertaining to demographics, presentation, radiological characteristics, management and outcome were collected. Cases were classified into spontaneous and barotraumatic PTX/PM and a between-group comparison was performed using Chi-square and $t$-test. A total of 45 cases (mean age: 53.2 years, $82 \%$ males) out of 8,294 confirmed COVID-19 patients developed PTX/PM, the calculated incidence being $0.54 \%$. 29 cases had spontaneous PTX/PM and the remaining 17 cases were attributed to barotrauma. The most common comorbidities were diabetes-mellitus $(65.3 \%)$ and hypertension (42.3\%). The majority of the cases had large PTX (62.1\%) with tension in 8 cases $(27.5 \%)$. There were predominant right-sided pneumothoraces and five were diagnosed with bronchopleural fistula. $37.7 \%$ of cases had associated subcutaneous emphysema. The median duration of PTX/PM from symptom onset was delayed at 22.5 and 17.6 days respectively. The mean CT severity score (CTSS) was 20.5 ( \pm 4.9 ) with fibrosis $(53.8 \%)$, bronchiectatic changes $(50 \%)$ and cystic-cavitary changes $(23 \%)$. There was no statistically significant difference between the spontaneous and barotrauma cohort. $71 \%$ of cases died and the majority belonged to the barotrauma cohort. It is imperative to consider the possibility of PTX/PM in patients having COVID-19, especially in those with deterioration in the disease course, both in spontaneously breathing and mechanically ventilated patients. These patients may also have a high incidence of death, reflecting the gravity of COVID-19.

Keywords Pneumothorax, pneumomediastinum, COVID-19

\section{Introduction}

The COVID-19 pandemic has resulted in significant mortality and morbidity around the globe. A varied number of manifestations and complications have been reported to be associated with the disease, however, the frequency of such manifestations is difficult to quantify (1). SARS-CoV-2 primarily affects the lung parenchyma with features ranging from mild selflimiting upper respiratory infection to critical acute respiratory distress syndrome (ARDS). A myriad of extra-parenchymal manifestations has been documented in the backdrop of COVID-19 such as pulmonary embolism, pleural effusion, empyema, cavitation and cyst formation (2-5). Pneumothorax (PTX) and pneumomediastinum (PM), defined as gas in the pleural cavity and the mediastinum respectively, has also been reported in association with COVID-19, although not as frequently as lung parenchymal involvement.

PTX and PM can be either arise spontaneously or due to barotrauma. Necrotizing pneumonia or lung parenchymal involvement, due to various aetiologies, 
can result in PTX/PM. The causes include commonly Pneumocystis jirovecii pneumonia (PCP), bacterial pneumonia caused by Staphylococci, Mycoplasma pneumoniae, Klebsiella and Pseudomonas spp., bronchiolitis obliterans organising pneumonia (BOOP) and sometimes by rarer organisms such as Herpes simplex, cytomegalovirus pneumoniae, Aspergillus and even influenzae virus (6-12). The previous Middle East respiratory syndrome (MERS) and severe acute respiratory syndrome coronavirus 1 (SARS-CoV-1) outbreaks have been associated with an increased incidence of PTX and PM (16.4\% and 1.7-11.6\%, respectively) associated with increased mortality (1315). Although several studies of PTX/PM in COVID-19 exist, there is sparse data on the incidence or frequency of occurrence, especially from the Indian subcontinent. In one of the earliest studies reporting pneumothoraces by Chen et al., only $\sim 1 \%$ (1/99 patients) had radiological evidence of PTX (16). In another study by Yang et al., out of 92 deceased COVID-19 patients, one (1.1\%) had a PTX (17). A recent systematic review by Chong et al. found the incidence of PTX to be $\sim 0.3 \%$ of hospitalised COVID-19 patients with rates increasing to $12.8-23.8 \%$ with $100 \%$ mortality in patients on invasive mechanical ventilation (18). The incidence of barotrauma has varied from $0-49 \%$ in traditional ARDS patients (19). Miro et al. reported a higher incidence of spontaneous PTX in patients with COVID-19 disease than among patients without COVID-19 disease (20). Similarly, McGuinness et al. reported a higher incidence of barotrauma among patients with COVID-19 acute respiratory distress syndrome in comparison to the historical controls with non-COVID-19 acute respiratory distress syndrome patients (21). Lemmers et al. found that the incidence of PTX was significantly greater in patients with COVID-19 ARDS despite the use of lung-protective ventilation, indicating that the frailty of the lung due to lung damage was contributing to the higher incidence of PTX in this patient population (22).

We present here a large cohort of PTX/PM from India, developing in admitted COVID-19 patients, and describe the demographic details, clinical features, management, risk factor for mortality and outcome of COVID-19 patients presenting with these entities.

\section{Materials and Methods}

This retrospective observational study was approved by the institutional ethics committee of the All India Institute of Medical Sciences (Institute Reference number IECPG/373/6/2021) and the need for patient consent was waived given the retrospective nature of the study. The authors used the electronic medical record (EMR) to attain clinical variables and admission details. All the relevant imaging studies were obtained by using the Picture Archiving and Communication System (PACS) and were reviewed by a pulmonologist and a radiologist independently (each with experience in the respective field of more than 10 years each). Only the cases where there was an agreement on diagnosis were included in this study.

\subsection{Subject population}

Case files of all patients aged $>18$ years with COVID-19 confirmed by RT-PCR or SARS-CoV-2 Antigen-RDT assay between $1^{\text {st }}$ April 2020 to $31^{\text {st }}$ May 2021 at our tertiary care facility were reviewed. Only those cases meeting the World Health Organisation (WHO) case definition of confirmed SARS-CoV-2 infection were included (23). Medical records reporting PTX and/or PM were reviewed. Cases with a history of iatrogenic PTX as a result of central venous catheter insertion or tracheal injury post-intubation were excluded.

\subsection{Clinical data collection}

To describe the characteristics of the cohort, demographic, clinical course and management details were obtained for each case and recorded in a predesigned proforma. Demographic parameters included age, sex, smoking status and underlying comorbidities. Details pertaining to COVID-19 included the initial COVID-19 severity classification at the time of presentation to our facility, and the severity at the time of developing PTX/PM, initial symptomatology of COVID-19 chiefly fever, cough, expectoration, shortness of breath, fatigue, myalgia, sore-throat, anosmia, ageusia and diarrhoea. Details about PTX/PM include the size of PTX, site of PTX (unilateral vs. bilateral and right vs. left-sided), associated $\mathrm{PM}$ and subcutaneous emphysema, day of onset of PTX/PM from COVID-19 symptom onset and the mode of oxygenation at the time of diagnosis of PTX/PM. Patients were subsequently divided into two groups for comparison spontaneous and barotraumatic PTX/PM. Any patient who developed PTX/PM at room air, facemask, nasal prong or high-frequency nasal cannula (HFNC) were considered to have secondary spontaneous PTX/PM, while patients on positive pressure ventilation viz. non-invasive ventilation mask (NIV) and invasive mechanical ventilation (IMV) resulting in PTX/PM were considered to have barotraumatic PTX/PM. Size of PTX was based on the 2003 British Thoracic Society (BTS) guideline (24) for classification of PTX based on the radiographic film. Small PTX: distance $<2 \mathrm{~cm}$ between the lung margin and the chest wall at the level of the hilum. Large PTX: distance $\geq 2 \mathrm{~cm}$ between the lung margin and the chest wall at the level of the hilum. Tension PTX: Presence of mid-line shift or presence of hemodynamic compromise due to the PTX. In cases of broncho-pleural fistula, Cerfolio's classification was used for grading (25). Details about the diagnosis, management were collected including modality of 
imaging used for diagnosis of PTX/PM, conservative versus pigtail insertion/water seal intercostal tube drainage for management of PTX/PM and final outcome of the patient, i.e., death or discharge.

\subsection{Imaging characteristics}

Chest radiograph imaging feature of all patients was analysed and was scored based on the 18-point Brixia scoring developed by Borghesi et al. (26) exclusively for COVID-19 patients. This was done to quantify the degree of lung involvement and its correlation to developing PTX/PM. HRCT chest was also evaluated whenever available and the findings were recorded into the following categories: CTSS, ground-glass opacification, consolidation, bronchiectasis, interlobular septal thickening, fibrosis, cysts and others.

\subsection{Statistical analysis}

STATA version 12.1 (StataCorp) was used for the statistical analyses in this study. Categorical variables were represented as frequency and percentages while continuous variables were represented as mean ( \pm standard deviation). Chi-square test and $t$-test/ Wilcoxon rank-sum test were applied to calculate statistical differences between categorical variables and continuous variables respectively. Survival data were used to generate Kaplan-Meier curves with STATA version 12.1 (StataCorp). Survival was compared using the log-rank test. A $p$-value of $<0.05$ was considered to assume statistical significance.

\section{Results}

From $1^{\text {st }}$ April 2020 to $31^{\text {st }}$ May 2021, a total of 45 patients were identified to have PTX/PM after reviewing the electronic database. During this study period, a total of 8,294 patients diagnosed with COVID-19 were admitted to our tertiary care centre which is dedicated to COVID-19 services. The calculated frequency was $0.54 \%$ (95\% CI: $0.4-0.73 \%$ ). A total of 16 patients had isolated PTX, 16 patients had isolated PM and 13 patients developed concurrent PTX with PM as shown in Table 1. The mean $( \pm \mathrm{SD})$ age of the cohort was $53.2 \pm 14.9$ years and the male to female ratio was 4.6:1.

Pre-existing comorbidities were present in $57.7 \%$ ( $n$ $=26)$ of the population with 12 cases $(46 \%)$ having more than one comorbidity. The most common comorbidity was diabetes mellitus $(65.3 \%)$ followed by hypertension $(42.3 \%)$, coronary artery disease and malignancy $(19.2 \%$ each), hypothyroidism and chronic kidney disease (7.6\% each) and hypertriglyceridemia (3.8\%). Three cases had a prior history of pulmonary tuberculosis. A total of 9 cases $(31 \%)$ were current or ex-smokers in this cohort. There was no significant statistical difference between the spontaneous and barotrauma group concerning comorbidities and smoking status.

Table 1. Baseline characteristics of COVID-19 patients with PTX and/or PM and comparison between patients developing spontaneous versus barotraumatic PTX/PM

\begin{tabular}{|c|c|c|c|c|}
\hline Variables & Total, $n(\%)$ & Spontaneous $n(\%)$ & Barotrauma $n(\%)$ & $p$-value \\
\hline Age in years ${ }^{a}$ & $53.28 \pm 14.9$ & $51.8 \pm 13.9$ & $55.5 \pm 16.5$ & \\
\hline Sex & & & & 0.98 \\
\hline Male & $37(82.2 \%)$ & $23(62.1 \%)$ & $14(37.8 \%)$ & \\
\hline Female & $8(17.7 \%)$ & $5(62.5 \%)$ & $3(37.5 \%)$ & \\
\hline \multicolumn{5}{|l|}{ Comorbidities $(n=26)$} \\
\hline Diabetes Mellitus & $17(65.3 \%)$ & $11(64.7 \%)$ & $6(35.2 \%)$ & 0.77 \\
\hline Hypertension & $11(42.3 \%)$ & $8(72.7 \%)$ & $3(27.2 \%)$ & 0.49 \\
\hline Coronary artery disease & $5(19.2 \%)$ & $3(60 \%)$ & $2(40 \%)$ & 1.00 \\
\hline Hypothyroidism & $2(7.6 \%)$ & $0(0 \%)$ & $2(100 \%)$ & 0.13 \\
\hline Malignancy & $5(19.2 \%)$ & $5(100 \%)$ & $0(0 \%)$ & 0.14 \\
\hline Chronic kidney disease & $2(7.6 \%)$ & $2(100 \%)$ & $0(0 \%)$ & 0.51 \\
\hline Hypertriglyceridemia & $1(3.8 \%)$ & $1(100 \%)$ & $0(0 \%)$ & 0.37 \\
\hline COPD/Asthma & $1(3.8 \%)$ & $0(0 \%)$ & $1(100 \%)$ & 0.37 \\
\hline \multicolumn{5}{|l|}{ Smoking status $(n=29)$} \\
\hline Smoker & $9(31 \%)$ & $7(77.7 \%)$ & $2(22.2 \%)$ & 0.43 \\
\hline \multicolumn{5}{|l|}{ Symptoms $(n=45)$} \\
\hline Fever & $32(71.1 \%)$ & $20(62.5 \%)$ & $12(37.5 \%)$ & 0.95 \\
\hline Dry cough & $21(46.6 \%)$ & $14(66.6 \%)$ & $7(33.3 \%)$ & 0.56 \\
\hline Expectoration & $4(8.8 \%)$ & $1(25 \%)$ & $3(75 \%)$ & 0.14 \\
\hline Shortness of breath & $40(88.8 \%)$ & $26(65 \%)$ & $14(35 \%)$ & 0.35 \\
\hline Fatigue & $3(6.6 \%)$ & $3(100 \%)$ & $0(0 \%)$ & 0.27 \\
\hline Myalgia & $3(6.6)$ & $2(66.6 \%)$ & $1(33.3 \%)$ & 1.00 \\
\hline Sore-throat & $7(15.5 \%)$ & $6(85.7 \%)$ & $1(14.2 \%)$ & 0.22 \\
\hline Diarrhoea & $2(4.4 \%)$ & $1(50 \%)$ & $1(50 \%)$ & 1.00 \\
\hline Nausea & $1(2.2 \%)$ & $1(100 \%)$ & $0(0 \%)$ & 1.00 \\
\hline Anosmia/Ageusia & $2(4.4 \%)$ & $1(50 \%)$ & $1(50 \%)$ & 1.00 \\
\hline
\end{tabular}

a: Mean \pm Standard Deviation. 
The most common presenting symptom at admission was shortness of breath (88\%) followed by fever $(71.1 \%)$, cough without expectoration $(46.6 \%)$, sore throat (15.5\%), cough with expectoration (8.8\%), fatigue and myalgia (both 6.6\%), diarrhoea and anosmia/ ageusia $(4.4 \%)$ and nausea $(2.2 \%)$. There was no significant statistical difference between the two groups (spontaneous and barotrauma induced) with regards to cough. Of the 29 cases with pneumothoraces, based on the BTS 2003 classification of size of PTX, 37.9\% ( $n$ $=11)$ had a small PTX and $62.1 \%(n=18)$ had large pneumothoraces of which $27.5 \%(n=8)$ had tension pneumothoraces. Five cases were diagnosed with bronchopleural fistula with three cases having grade 3 air leak based on Cerfolio classification. Majority of the cases had unilateral PTX $(86.2 \%, n=25)$, however $68 \%(n=16)$ had right-sided involvement. Associated subcutaneous emphysema was present in $37.7 \%(n=$ 17) cases with similar distribution between the two groups. The mean $( \pm \mathrm{SD})$ duration from symptom onset to developing PTX/PM was $22.5( \pm 11.5)$ days and $17.6( \pm 8.8)$ days respectively. The most common mode of oxygenation at the time of developing PTX/ $\mathrm{PM}$ in the spontaneous group was nasal prong/facemask $(57 \%, n=16)$ followed by HFNC $(28.5 \%, n=8)$ and room air $(14.2 \%, n=4)$. In the barotrauma patients, NIV $(58.8 \%, n=7)$ was the most common modality of respiratory support while the rest $(41 \%, n=7)$ were on invasive mechanical ventilation. The mean PEEP given in the barotrauma cohort was $8.9( \pm 1.4) \mathrm{cm}$ of $\mathrm{H}_{2} \mathrm{O}$. The radiological modality of PTX/PM diagnosis was chest radiograph in $75.5(n=34)$ and the rest were diagnosed based on chest computed tomography.
Immediate intercostal tube drainage or pigtail insertion was done in $57.7 \%(n=26)$ cases while the rest were managed conservatively. A conservative approach was taken in all of the cases of isolated PM (with or without subcutaneous emphysema) and three cases of small PTX without much symptomatology. In our cohort, $71 \%(n=32)$ of the cases died. The proportion of patients discharged was higher in the spontaneous group although it was not statistically significant ( $p$ value 0.08 ). The median duration of hospitalisation was $17.5( \pm 12.9)$ days. The details are enumerated in Table 2. KaplanMeier curve was plotted to determine the probability of survival between the PM cohort and the PTX with/ without PM cohort. Even though the overall survival duration was not significant between the two groups concerning days from the event (PM and/or PTX) to death, there was a trend towards significance ( $p$ value $=0.07$ ) showing an increased survival in patients with PM vs. PTX as shown in Figure 1A. On the other hand, overall survival was non-significant with regards to the time of COVID-19 symptom onset to developing PTX/ PM $(p$ value $=0.27)$ as shown in Figure 1B.

Table 3 demonstrates the radiological characterization of the cohort. The most common chest CT feature was ground-glass opacification $(82 \%)$ characteristic of COVID-19 pneumonia. Other principal findings were consolidation (50\%), fibrosis $(53.8 \%$ ) with interlobular septal thickening (42.3\%) and bronchiectatic changes (50\%). A significant number of cases had cystic changes (23\%) and lung cavitation, which is an unusual feature of COVID-19 infection and was present in 3 cases (11.5\%). The mean CT severity score was $20.5( \pm 4.9)$ which indicated that the majority of cases had severe

Table 2. Characteristics, management and outcome of PTX/PM in COVID-19 and comparison between patients developing spontaneous versus barotraumatic PTX/PM

\begin{tabular}{|c|c|c|c|c|}
\hline Parameters & Total, $n(\%)$ & Spontaneous, $n(\%)$ & Barotrauma, $n(\%)$ & $P$-value \\
\hline Isolated pneumothorax & $16(35.5 \%)$ & $11(68.7 \%)$ & $5(31.2 \%)$ & - \\
\hline Isolated pneumomediastinum & $16(35.5 \%)$ & $10(62.5 \%)$ & $6(37.5 \%)$ & \\
\hline Pneumothorax and pneumomediastinum & $13(28.9 \%)$ & $7(53.8 \%)$ & $6(46.1 \%)$ & \\
\hline Pneumothorax size $(n=29)$ & & & & 0.51 \\
\hline Small & $11(37.9)$ & $6(54.5)$ & $5(45.4)$ & \\
\hline Large/Tension & $18(62.1)$ & $12(66.6)$ & $6(33.3)$ & \\
\hline Site of pneumothorax $(n=29)$ & & & & 0.13 \\
\hline Unilateral & $25(86.2)$ & $17(68)$ & $8(32)$ & \\
\hline Right side & 17 & 12 & 5 & \\
\hline Bilateral & $4(13.7)$ & $1(25)$ & $3(75)$ & \\
\hline Associated Subcutaneous emphysema $(n=45)$ & $17(37.7)$ & $9(52.9)$ & $8(47.0)$ & 0.31 \\
\hline Mode of oxygenation $(n=45)$ & 45 & & & \\
\hline Room air & & $4(14.2)$ & 0 & \\
\hline Nasal prong/face mask & & $16(57.1)$ & 0 & \\
\hline HFNC & & $8(28.5)$ & 0 & \\
\hline NIV & & 0 & $10(58.8)$ & \\
\hline Invasive mechanical ventilation & & 0 & $7(41.1)$ & \\
\hline Management & & & & 0.91 \\
\hline Intercostal tube drain/pigtail & $26(57.7)$ & $16(61.5)$ & $10(38.4)$ & \\
\hline Conservative & $19(42.2)$ & $12(63.1)$ & $7(36.8)$ & \\
\hline Outcome & & & & 0.08 \\
\hline Death & $32(71)$ & $17(53.1)$ & $15(46.8)$ & \\
\hline Discharge & $13(28.9)$ & $11(84.6)$ & $2(15.3)$ & \\
\hline
\end{tabular}


COVID-19. For the evaluation of the chest radiograph, the Brixia scoring developed by Borghesi et al. (26) was utilised. The mean $( \pm$ SD) Brixia score for the patients was $3.275( \pm 2.59)$ and there was no statistical difference between patients who survived and patients who died.

\section{Discussion}

In our cohort of 8,294 hospitalized COVID-19 patients, the prevalence of PTX and/or PM was $0.54 \%(95 \%$ CI: $0.4-0.73 \%$ ), with prevalence of PTX and PM being both $0.2 \%$ (95\% CI: $0.1-0.3 \%$ ) and that of combined PTX/PM being 0.16\% (95\% CI: 0.08-0.3\%). This study
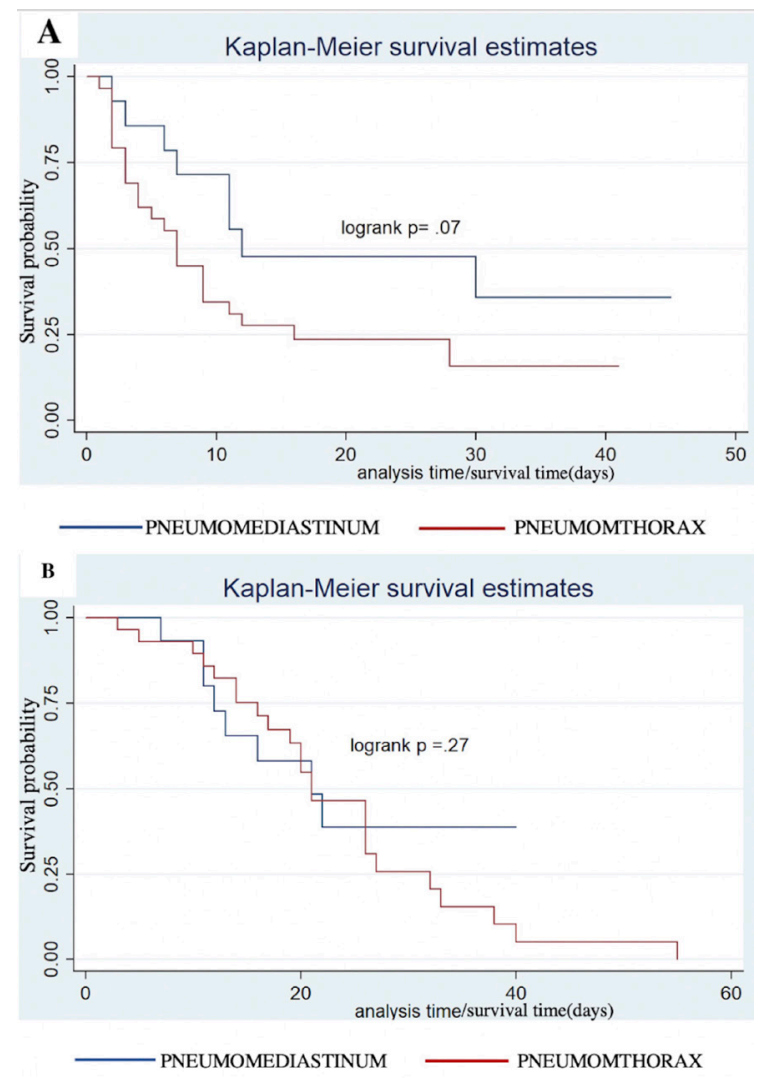

Figure 1. (A) Kaplan-Meier survival curve from the time of the event (PTX/PM) to time of death in days between PM (blue) and PTX (red). Log-rank test, $p=0.07$. (B) Kaplan-Meier survival curve from the time of the COVID-19 symptom onset to time of the event (PTX/PM) in days between PM (blue) and PTX (red). Log-rank test, $p=0.27$. pegs the prevalence of PTX and/or PM in COVID-19 patients reflecting on the frequency of occurrence of this serious complication against the backdrop of lung involvement by SARS-CoV-2. A systematic review, which did not include any Indian studies, had found the prevalence of PTX of $0.3 \%$ and $12.8-23.8 \%$ in hospitalized COVID-19 patients and those requiring invasive mechanical ventilation respectively (18). The incidence of spontaneous PTX/PM was $0.48 \%$ in our cohort, which was similar to the incidence of spontaneous PTX (0.66\%) reported from over 3,000 patients by Zantah et al. in their single centre study from the USA (27). However smaller studies had reported higher percentages $(\geq 1 \%)(17,28)$. The incidence of PTX associated with ARDS or mechanical ventilation in COVID-19 patients seems to be higher ranging from $10-56 \%(18,29)$. This study also provides a prevalence estimate of $0.2 \%$ of PM with or without PTX in COVID-19 patients, which is lower than that observed by Tacconi et al. (2.3\%) in a select group of patients (29). Spontaneous and barotrauma-related PTX and/or PM constituted $62 \%$ and $38 \%$ of the total 45 cases in our cohort. There were no significant differences between the spontaneous and barotraumarelated groups in terms of clinical parameters, radiological features or outcome.

The postulated mechanism for the development of PTX/PM is linked to the development of diffuse alveolar damage, cystic lesions due to fibro-inflammatory changes and fibrosis. Alveolar rupture, with or without the contribution of increased intrathoracic pressure during coughing or positive pressure ventilation, typically results in PTX or PM. PM and resulting subcutaneous emphysema, without concomitant significant PTX, can ensue due to the phenomenon of the Macklin effect (30). The pathologic features in the lungs of patients with COVID-19 pneumonia closely resemble those of SARS and MERS outbreaks. Histologic examinations have reported diffuse alveolar damage with fibromyxoid inflammatory exudates along with cystic lesions (31). In our cohort where CT imaging was available (26 cases), $53.8 \%$ of cases had evidence of fibrosis while $23 \%$ had cystic-cavitation changes, both of which are known contributing factors for the development of PTX/PM. The mean CTSS score for the cases was 20.5, indicating

Table 3. Radiological characteristics in COVID-19 patients with PTX/PM

\begin{tabular}{|c|c|c|c|c|}
\hline & Total, $n(\%)$ & Spontaneous, $n(\%)$ & Barotrauma, $n(\%)$ & $P$-value \\
\hline \multicolumn{5}{|l|}{ Radiological features $(n=26)$} \\
\hline Ground glass opacities & $22(84.0)$ & $17(77.2)$ & $5(22.7)$ & 0.28 \\
\hline Consolidation & $13(50.0)$ & $7(53.8)$ & $6(46.1)$ & 0.07 \\
\hline Bronchiectasis & $14(53.8)$ & $10(71.4)$ & $4(28.5)$ & 1.00 \\
\hline Fibrosis & $13(50)$ & $9(69.2)$ & $4(30.7)$ & 1.00 \\
\hline Interlobular septal thickening & $11(42.3)$ & $8(72.7)$ & $3(27.2)$ & 1.00 \\
\hline Cysts & $6(23.0)$ & $5(83.3)$ & $1(16.6)$ & 1.00 \\
\hline Cavitation & $3(11.5)$ & $1(33.3)$ & $2(60.6)$ & 0.16 \\
\hline
\end{tabular}


that the development of PTX/PM was related to the severity of lung involvement by COVID-19. The mean age of the cases was 53 years and the complication was more common in the male gender. Incidentally, men, as compared to women, are more likely to be afflicted by severe form of the COVID-19 infection as shown by a recent meta-analysis by Peckham et al. (32). Only one patient had underlying respiratory comorbidity $(3.8 \%)$ while significant smoking history was present in $31 \%$ indicating that COVID-19 was responsible for PTX/ $\mathrm{PM}$ in the vast majority. Brixia Scoring for the cases showed a mean value of 3.275 and it did not predict mortality.

An interesting observation made in our study was that right-sided PTX was more common than the left side (Right: left $=2: 1$ ). A similar observation was found by Miro et al. who reported that COVID-19-related PTX was 3.85-fold more likely to occur on the right side and at higher frequency $(81.1 \%$ versus $52.7 \% ; p<0.001)$ as compared to non-COVID-19 patients (20). The likely explanation for such observation may be a heightened degree of involvement of the right lung vis-à-vis the left lung, as reported by previous research (33-35). There could be several reasons for this, including the larger size and number of segments of the right lung, as well as the larger diameter of the right main bronchus leading to greater delivery of viral particles to the right lung causing relatively more damage (34).

Another interesting finding in this study was the median duration of PTX/PM from symptom onset which was 22.5 and 17.6 days respectively. Similarly, delayed onset of PTX/PM has been seen in most of the previous larger studies. The late onset of this complication suggests a sustained period of lung inflammation with extensive parenchymal injury followed by fibrosis and cyst formation in the late phase of COVID-19 pneumonia. The late finding is also supported by the typical radiologic findings in COVID-19 patients (36) comprising of inflammation of the lung parenchyma that predominantly affects the peripheries progressing to consolidation and eventually involves the overlying pleura leading to various pleural manifestations such as PTX and pleural effusion. The majority of the cases in our study died $(71 \%)$ and most of the cases who survived belonged to the spontaneous group as opposed to the barotrauma group even though there was no statistical significance. Such high mortality is comparable to a recent systematic review by Chong et al. reporting an overall mortality rate of $74.2 \%(18)$. The mortality rate of severe COVID-19 is known to be very high and our study demonstrates that severe COVID-19 patients with PTX/PM have a very prognosis. With regards to isolated $\mathrm{PM}$, the mortality rate was $0.56 \%$ which was considerable drawing to the conclusion that not only PTX but also PM is a significant predictor of poor outcome in patients with COVID-19. KaplanMeier survival curve depicted in Figures 1A and 1B shows that the PM cohort of cases had better chances of survival as compared to cases with PTX. The possible explanation lies in the fact that PTX results in decreased lung volume leading to further compromise of lung function in the already poor compliant lung parenchyma culminating in a poor outcome. On the other hand, pneumomeiastinum doesn't result in compromise of lung function unless the size is massive with all the cases undergoing conservative management possibly explaining the improved survival.

This study involves the largest cohort of patients from the Indian subcontinent concerning the prevalence, clinico-radiological characteristics and outcome of PTX and/or PM in COVID-19 patients. However, this study is not without limitations. The most significant one was that it was a retrospective study from a single tertiary centre, with potential risks of information and selection biases.

\section{Conclusion}

It is thus imperative to consider the possibility of PTX and/or PM in patients having COVID-19, especially in those with deterioration in the disease course, both in spontaneously breathing as well as mechanically ventilated patients. These patients may also have a high incidence of death, apparently reflecting the gravity of COVID-19 disease.

\section{Funding: None.}

Conflict of Interest: The authors have no conflicts of interest to disclose.

\section{References}

1. Gupta A, Madhavan MV, Sehgal K, et al. Extrapulmonary manifestations of COVID-19. Nat Med. 2020; 26:10171032.

2. Porcel JM. Pleural diseases and COVID-19: ubi fumus, ibi ignis. Eur Respir J. 2020; 56:2003308.

3. Bompard F, Monnier H, Saab I, Tordjman M, Abdoul H, Fournier L, Sanchez O, Lorut C, Chassagnon G, Revel MP. Pulmonary embolism in patients with COVID-19 pneumonia. Eur Respir J. 2020; 56:2001365.

4. Aayilliath KA, Singh K, Ray A, Wig N. Platypnoeaorthodeoxia syndrome in COVID-19. BMJ Case Rep. 2021; 14:e243016.

5. Sarda R, Swain S, Ray A, Wig N. COVID-19 associated Mucormycosis and evolving evidence. QJM. 2021.

6. Al-Ghanem S, Al-Jahdali H, Bamefleh H, Khan AN. Bronchiolitis obliterans organizing pneumonia: pathogenesis, clinical features, imaging and therapy review. Ann Thorac Med. 2008; 3:67-75.

7. Chen $\mathrm{CH}$, Liao WC, Liu YH, Chen WC, Hsia TC, Hsu WH, Shih CM, Tu CY. Secondary spontaneous pneumothorax: which associated conditions benefit from pigtail catheter treatment? Am J Emerg Med. 2012; 30:4550 .

8. Coker RJ, Moss F, Peters B, McCarty M, Nieman R, Claydon E, Mitchell D, Harris JR. Pneumothorax in 
patients with AIDS. Respir Med. 1993; 87:43-47.

9. López-Rivera F, Colón Rivera X, González Monroig HA, Garcia Puebla J. Pneumomediastinum and pneumothorax associated with herpes simplex virus (HSV) pneumonia. Am J Case Rep. 2018; 19:109-113.

10. Olliff JF, Williams MP. Radiological appearances of cytomegalovirus infections. Clin Radiol. 1989; 40:463467.

11. Sepkowitz KA, Telzak EE, Gold JW, Bernard EM, Blum S, Carrow M, Dickmeyer M, Armstrong D. Pneumothorax in AIDS. Ann Intern Med. 1991; 114:455-459.

12. Suri T, Makkar N, Ray A, Sood R. A unique case of hydropneumothorax in allergic bronchopulmonary aspergillosis. Med Mycol Case Rep. 2019; 25:29-31.

13. Chu CM, Leung YY, Hui JY, Hung IF, Chan VL, Leung WS, Law KI, Chan CS, Chan KS, Yuen KY. Spontaneous pneumomediastinum in patients with severe acute respiratory syndrome. Eur Respir J. 2004; 23:802-804.

14. Das KM, Lee EY, Al Jawder SE, Enani MA, Singh R, Skakni L, Al-Nakshabandi N, AlDossari K, Larsson SG. Acute middle east respiratory syndrome coronavirus: Temporal lung changes observed on the chest radiographs of 55 patients. AJR Am J Roentgenol. 2015; 205:W267274.

15. Sihoe AD, Wong RH, Lee AT, Lau LS, Leung NY, Law KI, Yim AP. Severe acute respiratory syndrome complicated by spontaneous pneumothorax. Chest. 2004; 125:2345-2351.

16. Chen N, Zhou M, Dong X, Qu J, Gong F, Han Y, Qiu Y, Wang J, Liu Y, Wei Y, Xia J, Yu T, Zhang X, Zhang L. Epidemiological and clinical characteristics of 99 cases of 2019 novel coronavirus pneumonia in Wuhan, China: a descriptive study. Lancet. 2020; 395:507-513.

17. Yang F, Shi S, Zhu J, Shi J, Dai K, Chen X. Analysis of 92 deceased patients with COVID-19. J Med Virol. 2020; 92:2511-2515.

18. Chong WH, Saha BK, Hu K, Chopra A. The incidence, clinical characteristics, and outcomes of pneumothorax in hospitalized COVID-19 patients: A systematic review. Heart Lung. 2021; 50:599-608.

19. Boussarsar M, Thierry G, Jaber S, Roudot-Thoraval F, Lemaire F, Brochard L. Relationship between ventilatory settings and barotrauma in the acute respiratory distress syndrome. Intensive Care Med. 2002; 28:406-413.

20. Miró Ò, Llorens P, Jiménez S, et al. Frequency, risk factors, clinical characteristics, and outcomes of spontaneous pneumothorax in patients with coronavirus disease 2019: A case-control, emergency medicine-based multicenter study. Chest. 2021; 159:1241-1255.

21. McGuinness G, Zhan C, Rosenberg N, Azour L, Wickstrom M, Mason DM, Thomas KM, Moore WH. Increased incidence of barotrauma in patients with COVID-19 on invasive mechanical ventilation. Radiology. 2020; 297:E252-e262.

22. Lemmers DHL, Abu Hilal M, Bnà C, Prezioso C, Cavallo E, Nencini N, Crisci S, Fusina F, Natalini G. Pneumomediastinum and subcutaneous emphysema in COVID-19: barotrauma or lung frailty? ERJ Open Res. 2020; 6:00385-2020.

23. WHO. COVID-19 case definition. https://www.who.int/ publications/i/item/WHO-2019-nCoV-Surveillance_Case_
Definition-2020.2 (accessd August 20, 2021).

24. Henry M, Arnold T, Harvey J. BTS guidelines for the management of spontaneous pneumothorax. Thorax. 2003; 58 (Suppl 2):ii39-52.

25. Cerfolio RJ. Recent advances in the treatment of air leaks. Curr Opin Pulm Med. 2005; 11:319-323.

26. Borghesi A, Zigliani A, Masciullo R, Golemi S, Maculotti P, Farina D, Maroldi R. Radiographic severity index in COVID-19 pneumonia: relationship to age and sex in 783 Italian patients. Radiol Med. 2020; 125:461-464.

27. Zantah M, Dominguez Castillo E, Townsend R, Dikengil F, Criner GJ. Pneumothorax in COVID-19 diseaseincidence and clinical characteristics. Respir Res. 2020; 21:236.

28. Wang XH, Duan J, Han X, Liu X, Zhou J, Wang X, Zhu L, Mou H, Guo S. High incidence and mortality of pneumothorax in critically Ill patients with COVID-19. Heart Lung. 2021; 50:37-43.

29. Tacconi F, Rogliani P, Leonardis F, Sarmati L, Fabbi E, De Carolis G, La Rocca E, Vanni G, Ambrogi V. Incidence of pneumomediastinum in COVID-19: A single-center comparison between $1^{\text {st }}$ and $2^{\text {nd }}$ wave. Respir Investig. 2021; 59:661-665.

30. Ray A. A letter in response to recurrent subcutaneous emphysema in a treated tuberculosis patient: Is there any association? Lung India. 2014; 31:312-314.

31. Xu Z, Shi L, Wang Y, et al. Pathological findings of COVID-19 associated with acute respiratory distress syndrome. Lancet Respir Med. 2020; 8:420-422.

32. Peckham H, de Gruijter NM, Raine C, Radziszewska A, Ciurtin C, Wedderburn LR, Rosser EC, Webb K, Deakin CT. Male sex identified by global COVID-19 metaanalysis as a risk factor for death and ITU admission. Nat Commun. 2020; 11:6317.

33. Haseli S, Khalili N, Bakhshayeshkaram M, Sanei Taheri M, Moharramzad Y. Lobar distribution of COVID-19 pneumonia based on chest computed tomography findings; A retrospective study. Arch Acad Emerg Med. 2020; 8:e55.

34. Nagra D, Russell M, Yates M, Galloway J, Barker R, Desai SR, Norton S. COVID-19: opacification score is higher in the right lung and right lung involvement is a better predictor of ICU admission. Eur Respir J. 2020; 56:2002340.

35. Yang R, Li X, Liu H, Zhen Y, Zhang X, Xiong Q, Luo Y, Gao C, Zeng W. Chest CT severity score: An imaging tool for assessing severe COVID-19. Radiol Cardiothorac Imaging. 2020; 2:e200047.

36. Shi H, Han X, Jiang N, Cao Y, Alwalid O, Gu J, Fan Y, Zheng C. Radiological findings from 81 patients with COVID-19 pneumonia in Wuhan, China: a descriptive study. Lancet Infect Dis. 2020; 20:425-434.

Received December 2, 2021; Revised December 22, 2021; Accepted December 27, 2021

*Address correspondence to:

Animesh Ray, Department of Medicine, All India Institute of Medical Sciences, Room no: 3070A, 3rd Floor Teaching Block, Ansarinagar, New Delhi 110029, India.

E-mail: doctoranimeshray@gmail.com 